\title{
Gas Coupled Polymeric Capacitive Transducers via Pad Printing
}

\author{
Richard L. O'Leary \\ Centre for Ultrasonic Engineering, Dept. of Electronic and Electrical Eng., \\ University of Strathclyde \\ Glasgow, Scotland, UK \\ richard.o-leary@strath.ac.uk
}

\begin{abstract}
Micropatterning of polymer substrates has been shown to be effective methodology for the manufacture of capacitive transducers. The method involves creating a positive mask of sessile liquid droplets on a polymer substrate. The droplets define the cavity dimensions and spatial distribution, a subsequent processing stage independently defines the cavity depth. Droplets can be defined in a variety of ways - this paper explores the patterning the droplets, and hence cavities, on the polymer substrate via a pad-printing technique. The printing pad is manufactured using $3 D$ printing technique, an example pad is illustrated in Figure 1 - essentially the pad comprises an array of styli. The lateral dimensions and spatial distribution of the styli are replicated via the pattern of deposited droplets on the polymer substrate. The morphology of the styli tips has been explored - planar tips were found to droplet patterns with the highest fidelity. Single element air coupled devices operating with a nominal centre frequency of $500 \mathrm{kHz}$ have been constructed and evaluated experimentally. Transducer bandwidths in excess of $100 \%$ were observed with two-way insertion loss of $60 \mathrm{~dB}$ being typical.
\end{abstract}

Keywords - capacitive transducer; rapid prototyping; air coupled ultrasound

\section{INTRODUCTION}

Capacitive micromachined ultrasonic transducers (cMUT) are a viable alternative to piezoelectric devices in a number of key areas of ultrasound technology. cMUT devices offer a number of benefits including, short pulse length, wide operational bandwidth and can be configured to offer enhanced electro-mechanical coupling to gaseous media.

cMUT devices are typically manufactured via formation of a regular array of uniformly dimensioned cavities in a substrate. A membrane is positioned above each cavity transmission and reception of ultrasound is effected via controlled electrical or mechanical stimulus of the membrane, respectively. The vibrational behavior and operational frequency of the device is governed by a number of key design parameters: such as, the cavity dimensions and spatial distribution and the mechanical properties of the membrane.

The manufacturing of contemporary cMUT typically employs subtractive processes such as micromachining of silicon in order to form the cavities of the device [1], [2]. Such methods allow for the manufacture of large numbers of devices on a single silicon wafer with high yield rates are reported. Additive manufacturing processes, such as micro- stereolithigraphy (MSL) have been assessed for efficacy for cMUT manufacture [3], [4]. Whilst MSL is capable for the construction of the majority of the components of the cMUT device, additional processes are required to apply electrodes. Typical MSL constructed devices tend to be limited to low operating frequencies $<200 \mathrm{kHz}$ [3], [4] - a consequence of the available voxel size in the MSL process. More recently, the author has reported a new process for the manufacture of cavities within cMUT devices [5], [6]. Deposition of a positive mask onto a polymer substrate which is subsequently exposed to a vapour of solvent, which is absorbed into the substrate allows for controlled formation of cavities. In the work reported to date, each individual cavity is formed by a sessile liquid droplet deposited onto a polymer substrate. Aerosol deposition results in spatially and dimensionally random cavities, the use of a CNC equipped with a piezoelectric droplet generator affords creating spatial and dimensional control. The latter process is serial in nature, requiring long processing times for large device apertures.

In order to address the long processing times, this paper investigates the use of pad printing as a method of depositing the sessile droplets onto the polymer substrate. A print-pad comprises an array of styli. The layout of the styli corresponds to the required diameter and spatial distribution of the droplets array in order to create cavities for a given operational frequency. The use of the pad-printing approach affords a single operation for the deposition of the entire array of droplets required to form the cavities of a device.

In the following section, the manufacturing process will be described. This is followed by the experimental assessment of the fidelity of droplet patterns for different styli morphologies. Finally, the manufacture and experimental assessment of a pair of air-coupled devices is presented.

\section{Methodology}

Prior to investigating a specific cavity design for a given transducer operating frequency, an assessment of pad morphology was undertaken in order to assess the potential for pad printing to accurately reproduce a given pattern. Matlab (Mathworks, Natick, MA) was employed to create the stereolithography (STL) file required for printing. A 3- 
dimensional mesh was constructed based on a circular aperture populated with an array of cylindrical styli of the desired diameter and spatial distribution. The mesh was then converted to the STL file format [8]. An example pad design comprising cylindrical styli with planar is shown in Fig. 1. In addition to the planar tipped pad, designs comprising styli tips of a conical morphology were investigated, both convex and concave designs were assessed - in both cases the cone angle was $30^{\circ}$ to the vertical axis.

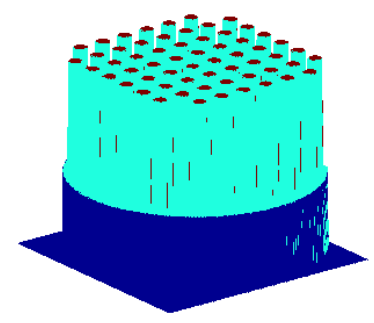

Fig. 1. Example pad design

The pads were printed using a Pico Plus 27 (Asiga, Anaheim Hills, CA) 3D printing system. The system has a maximum build volume of $35 \times 21.8 \times 76 \mathrm{~mm}$ and resolution of $27 \mu \mathrm{m}$ in both $\mathrm{x}-$ and $\mathrm{y}$-axes and $10 \mu \mathrm{m}$ resolution in the $\mathrm{z}$-axis. Once printed the pads were post-cured under UV light and then tested.

\section{A. Assesment of print pad styli morphology}

Polystyrene sheet, $3 \mathrm{~mm}$ in thickness (Amari Plastics, Glasgow), was cut to the desired dimensions and cleaned via sonication in methanol (Sigma Aldrich, Dorset, UK) and air dried at room temperature. An aliquot of liquid polyethylene glycol (PEG) (Sigma Aldrich, Dorset, UK) was spread evenly on a sheet of acetate film, each pad produced via MSL was placed onto the film of PEG such that tips of the styli would each acquire a small volume of PEG. The pad was then transferred to the sample of polystyrene, the pad was removed and the resulting pattern of droplets was assessed using a microscope coupled camera - Fig. 2 details photomicrographs for each styli type.

Each of the photomicrographs shown in Fig. 2 details a small subset of the deposed droplets for each of the styli designs. However the images shown are representative of the performance of each design- in each case the pad design is $500 \mu \mathrm{m}$ diameter on a $2 \mathrm{~mm}$ pitch. Considering the convex case, shown in Fig. 2(b), it is clear that the conical tips have not faithfully reproduced the desired patter, droplet are not at their expected locations. Moreover, the droplets that are on the polystyrene sheet do not exhibit the required circular morphology. The styli with the concave tips, shown in Fig. 2(c) has more successfully reproduced the desired spatial distribution, however the droplets are not circular. This is expected to be a result of the increased surface area of the concave tips offering enhanced wetting to the PEG fluid and as pad it removed causing distortion of the droplets. The pattern resulting from the planar tipped styli, shown in Fig. 2(a) offers the best performance in terms of spatial distribution of circular droplets. As a result of this assessment planar tipped styli were used in the manufacture of the pads in order to create specific transducer designs.

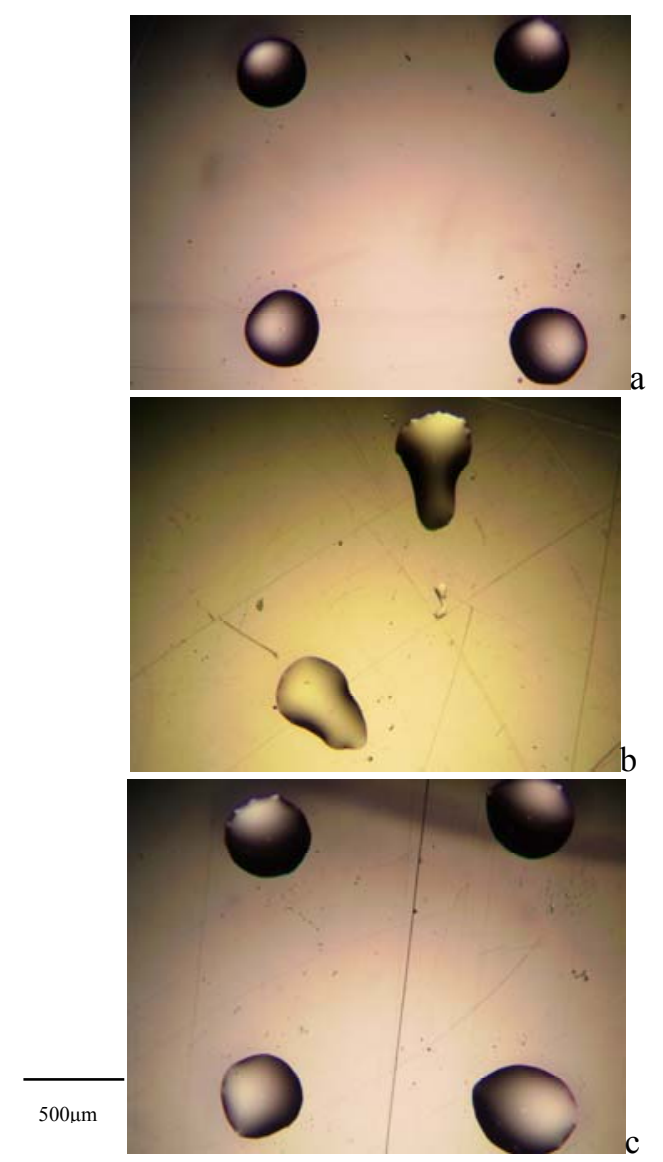

Fig. 2. Photomicrographs of pad printed pattern of droplets on polymer substrate: (a), pad with planat styli tip, (b) pad with convex tips and (c) pad with concave tips.

\section{TRANSDUCER MANUFACTURE}

Previous work by the author has indicated that for aircoupled operation, an orthogonal layout of $700 \mathrm{~nm}$ deep, $500 \mu \mathrm{m}$ diameter cavities on a $1000 \mu \mathrm{m}$ pitch will result in a $500 \mathrm{kHz}$ operation in air [6]. Matlab was used to create the design, a $20 \mathrm{~mm}$ circular aperture comprising $500 \mu \mathrm{m}$ diameter planar tipped styli on a $1000 \mu \mathrm{m}$ pitch. The deposition process was repeated as previously described, Fig. 3 details a images of the droplet pattern produced on a polystyrene substrate - it is clear that the pattern is faithfully reproduced. Once the droplets of PEG had been deposited onto the polystyrene the samples were exposed to a vapor of toluene. Polystyrene absorbs toluene causing it to swell, however regions of polystyrene under the deposited droplets do not absorb toluene and hence the do not swell thus forming small cavities. The period of time the sample is exposed to the toluene vapor governs the extent of the swelling and hence depth of the cavity [5], [6]. After exposure to toluene vapor, the sample is air dried at $20^{\circ} \mathrm{C}$ and 
then washed by sonication in methanol to remove the PEG droplets and further air-drying at $20^{\circ} \mathrm{C}$. In order to manufacture transducers, the polystyrene substrate is then electroded on all faces with silver using an electroless process with a tin sensitizer [7].
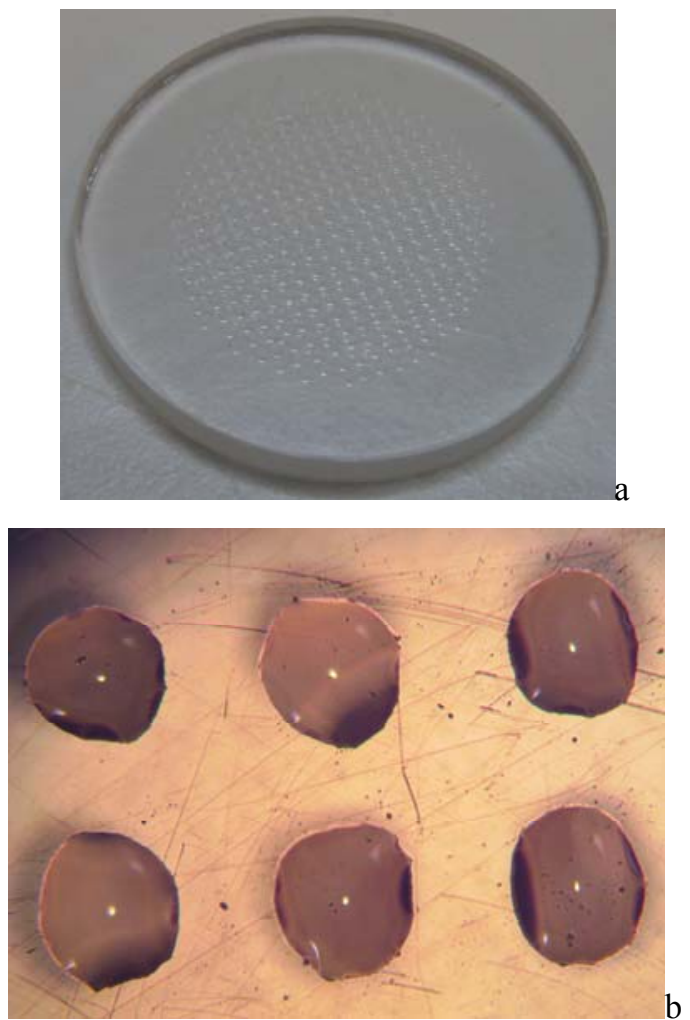

Fig. 3. Images of the droplet pattern deposited on polystyrene substrate: (a) entire aperture and (b) a subset of droplets

The micro-patterned polystyrene was then fitted with Kapton membrane (Goodfellow, Huntingdon, UK) the outer face of the dielectric membrane has a sputtered aluminum electrode. Fig. 4 details a photograph of an example device once packaged with the front-face membrane

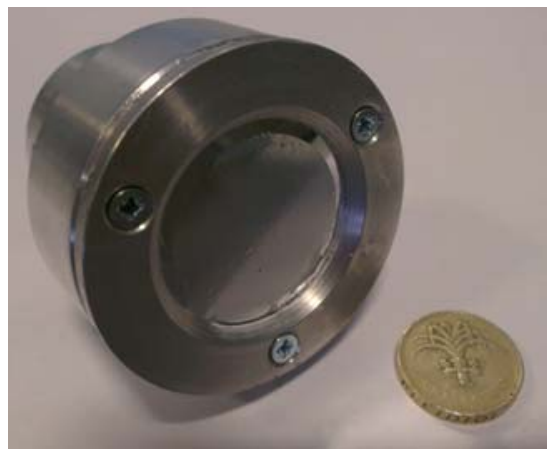

Fig. 4. Photograph of an example device.

\section{AIR-COUPLED TRANSDUCER PERFORMANCE}

A pair of identical devices was manufactured using the methodology. Performance of the devices was assessed using air coupled pulse echo response from a $50 \mathrm{~mm}$ glass block. In each case a $200 \mathrm{~V}$ bias supplied from a Brandenburg 475R with a Panametrics pulser-receiver model 5052PR being used to excite the membrane and amplify the resultant return echo. Fig. 5
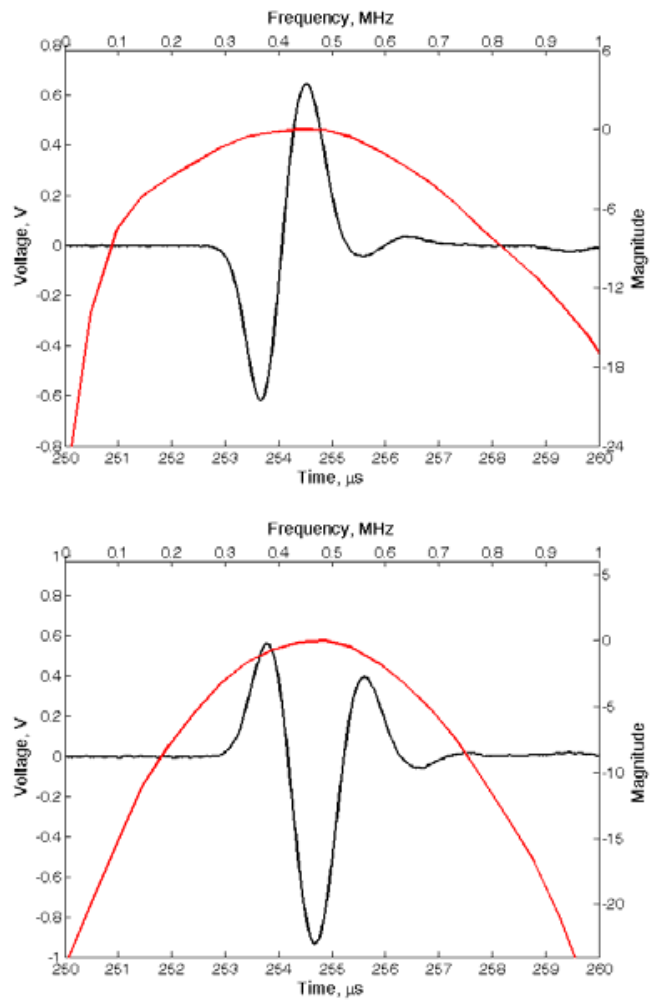

Fig. 5. Pulse echo response of each device

It is clear that both devices are capable of generating ultrasound in the desired frequency range. In order to further investigate their potential, pitch-catch performance was undertaken.

To assess the insertion loss of the pair of devices operating in air, a $10 \mathrm{~V}_{\mathrm{pp}}$, 3-cycle tone burst at the centre frequency of the devices was used to excite the transmitter; the data acquired at the receiver is shown in Fig. 6 . The voltage amplitude of the receive signal was found to be $10.9 \mathrm{mV}_{\mathrm{pp}}$ giving an insertion loss of $-59 \mathrm{~dB}$.

Finally laser vibrometry was performed using an OFV 056 (Polytec, Germany) to determine the surface dilation of the membrane when the device was subject to continuous wave excitation at the centre frequency. The magnitude and phase of the surface dilation for device one of the devices is shown in Fig. 7. It is clear that the individual cavities of the device are vibrating in phase.

For each of the measured transducer metrics, performance was found to be comparable to equivalent devices manufactured by alternate methodology [5], [6]. 


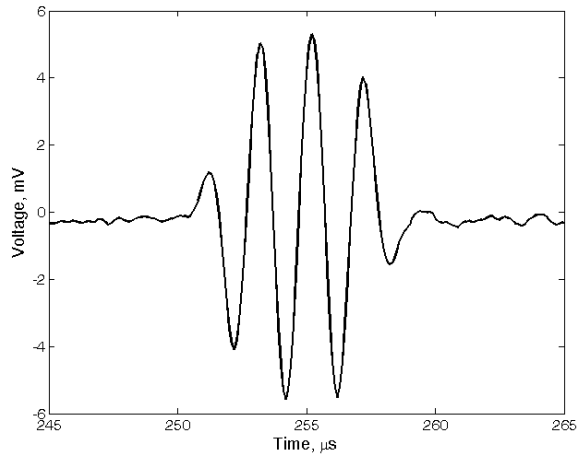

Fig. 6. Pitch catch behaviour of the pair of devices operating in air
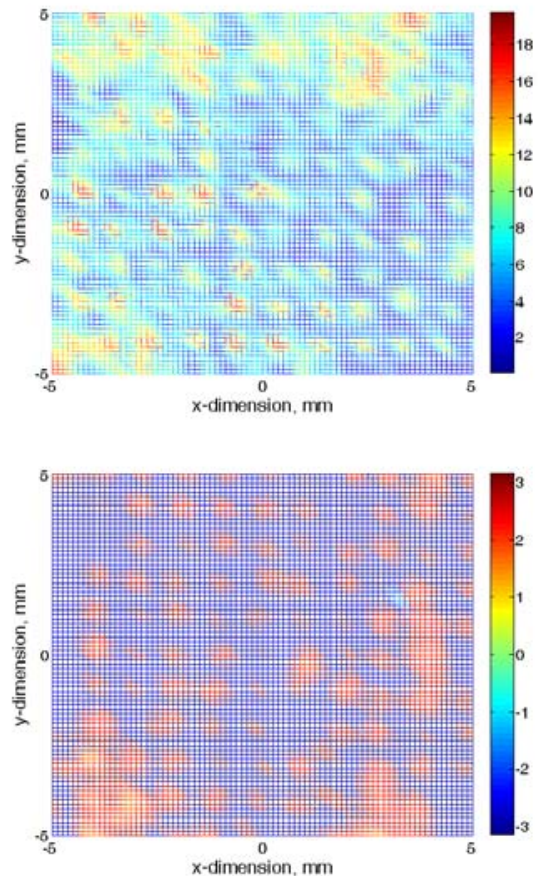

Fig. 7. Laser vibrometer determined surafce dilation, upper plot dialtion magnitude and lower plot is dilation phase

\section{CONCLUDING REMARKS}

This paper has explored the possibility of using a padprinting methodology for the production cMUT type aircoupled transducers. Printing pads manufactured using microstereolithography have been used to deposit an array of droplets onto a polymer substrate, the polymer substrate then undergoes further processing in order to manufacture a cMUT. Pads comprising planar tips were found to reproduce the desired droplet pattern with an appropriate level of fidelity.

A pair of devices was manufactured and assessed experimentally. Each device has equivalent operational characteristics with a nominal centre frequency of $500 \mathrm{kHz}$. The performance of the pad printed devices was found to be equivalent devices produced via the serial route using a droplet generator to deposit the array of individual drops [5], [6].

Future work will focus on extending the upper operational frequency beyond $1 \mathrm{MHz}$ and investigate the encapsulation of the devices for operation in liquid media.

\section{ACKNOWLEDGEMENTS}

The author would like to acknowledge Dr Francesco Guarato of the Centre for Ultrasonic Engineering at the University of Strathclyde for his diligence in preparing the MSL prepared samples.

\section{REFERENCES}

[1] D.W. Schindel, et al IEEE Trans Ultrasonics, Ferroelectrics and Frequency Control Vol 42, No 1, pp42-50 (1995)

[2] M.I. Haller, \& B.T. Khuri-Yakub, IEEE Transaction on UFFC, 1996, 43(1): p. 1-6

[3] K.S. Ho, R.J. Bradley, D.R. Billson and D.A. Hutchins, Ultrasonics. 48,1-5 (2008).

[4] S. B. Abdul Hamid, Ph.D. Thesis, University of Strathclyde, Glasgow, 2013

[5] R.L. O'Leary et al Proc IEEE IUS 2013, pp1113-16 doi 10.1109/ULTSYM.2013.0285

[6] R.L. O’Leary \& G. Harvey, Proc IEEE IUS 2014, pp177-80 doi 10.1109/ULTSYM.2014.0045

[7] G. Müller \& D.W. Baudrand ISBN 9780852180389

[8] B.Macdonald, http://www.mathworks.com/matlabcentral/fileexchange/4512-surf2stl, accessed 07 October 2015 